\title{
Regulating Heterogeneous Utilities: A New Latent Class Approach with Application to the Norwegian Electricity Distribution Networks
}

\author{
Luis Orea* and Tooraj Jamasb**
}

\begin{abstract}
Since the 1990s, electricity distribution networks in many countries have been subject to incentive regulation. The sector regulators aim to identify the best performing utilities as frontier firms to determine the relative efficiency of firms. This paper develops a nested latent class (NLC) model approach where unobserved differences in firm performance are modelled using two 'zero inefficiency stochastic frontier' (ZISF) models nested in a 'latent class stochastic frontier' (LCSF) model. This captures the unobserved differences due to technology or environmental conditions. A Monte Carlo simulation suggests that the proposed model does not suffer from identification problems. We illustrate the proposed model with an application to Norwegian distribution network utilities for the period 2004-2011. We find that the efficiency scores in both LCSF and ZISF models are biased, and some firms in the ZISF model are wrongly labelled as inefficient. Conversely, inefficient firms may be wrongly labelled as being fully efficient by the ZISF model.
\end{abstract}

Keywords: Latent class models, Environmental conditions, Electricity distribution, Reference networks

http://dx.doi.org/10.5547/01956574.38.4.lore

\section{INTRODUCTION}

Since the worldwide reform of electricity sector in the 1990s, many network utilities are incentive regulated. The reform trend coincided with some significant contributions to regulatory economics (Shleifer, 1985; Laffont and Tirole, 1993). The aim is to provide natural monopoly firms with incentives to improve their operating and investment efficiency and to ensure that consumers benefit from the gains. The main methods used to achieve these objectives are incentive mechanisms, which provide the firms with financial rewards and penalties linked to their performance (Joskow, 2008).

In many instances, the regulators aim to measure the firms' relative efficiency against best practice and performance using parametric and non-parametric techniques (see Haney and Pollitt, 2013). However, the sector regulators normally identify more than one efficient benchmark firm as they have a large and diverse number of utilities to choose from in the sector. Statistical benchmarking methods have been used to determine the relative efficiency of individual firms' operating

* Oviedo Efficiency Group, University of Oviedo, Spain. E-mail: lorea@uniovi.es.

** Corresponding author. Durham University Business School, Mill Hill Lane, Durham DH1 3LB, United Kingdom.

E-mail: tooraj.jamasb@durham.ac.uk. 
costs and service quality compared to and efficient frontier of their peers. ${ }^{1}$ The efficiency estimates are based on measuring the gap between the actual cost of the firm (production) and an optimal point on the cost (production) frontier, which is estimated from the available dataset.

The methodology developed in the paper is motivated by theoretical as well as empirical regulatory issues. First, since the adoption of efficiency benchmarking for incentive regulation in the 1990s, the expectation was that these methods would reduce the efficiency gap in the sector and that over time the regulation would transit towards a yardstick regulatory model using average sector performance as a benchmark. However, although average efficiency among firms has improved, efficiency gaps still persist among firms. Part of this observed inefficiency is most likely to have been caused by an inadequate control of the firms' technological heterogeneity.

Second, whether or how to account for heterogeneity, has, from the start, been a contentious issue between the regulators and the firms. As regulators reward or penalise firms in line with their respective (in)efficiency levels, the reliability of these scores is crucial for the fairness and effectiveness of the regulatory framework. Errors in identifying the correct benchmark firms or in the measurement of firm's efficiency have also resulted in significant financial implications for the less efficient firms against which they are compared. Obtaining reliable measures of firms' inefficiency requires controlling for the contextual factors under the influence of which each utility operates. ${ }^{2}$

In many countries, utilities have argued against the unfair effects of failing to account for the heterogeneity existing among firms. In the UK, the heterogeneity present in distribution networks has been addressed through some post-measurement adjustments to utility costs. In Germany, network utilities with fewer than 100,000 customers are exempted from benchmarking by the national regulator. In Brazil, the regulator separates the distribution networks into two size groups (small and large), dependent upon the units of energy distributed and using 1TWh as the dividing line. In Norway, the regulator uses some geographic and weather variables in the benchmarking models and also separates the regional mid-voltage networks from low-voltage distribution utilities in benchmarking models. In Chile, the regulator has used six different reference norm firms to account for the heterogeneity among the real firms. In Sweden, the larger networks strongly argued that the norm models used by the regulator proved disadvantageous to large networks. Given this context, methodologies designed to improve the accuracy of benchmarking methods, which are at the same time sensitive to technological and environmental heterogeneity can also help in deciding as to the true efficiency gap of a sector and the correct timing for moving to the yardstick regulation stage.

Recently, latent class stochastic frontier (hereafter LCSF) models that combine the stochastic frontier approach with a latent class structure have appeared in efficiency analysis literature to account for technological heterogeneity among firms. Latent class models, also called finite mixture models, have been used in several fields of research (see Orea and Kumbhakar, 2004; Greene, 2005, for some applications). A conventional LCSF model assumes there is a finite number of technologies (classes) underlying the data and allocates probabilistically each firm in the sample to a particular technology. Once the benchmark technology of each firm is identified, its inefficiency

1. Jamasb and Pollitt (2001) review the most commonly used approaches and provide a survey of benchmarking studies applied mainly in OECD countries. For a recent review of the applied literature on regulation of electricity distribution networks see, e.g., Kuosmanen (2012).

2. The inclusion of environmental variables (also referred to as contextual variables or z-variables) in the model is contentious in the literature on efficiency analysis and has generated the development of several models (for a review of this topic in Stochastic Frontier Analysis (SFA) and Data Envelopment Analysis (DEA) see, e.g., Johnson and Kuosmanen, 2012, and Llorca et al., 2014).

Copyright (C) 2017 by the IAEE. All rights reserved. 
in relation to that benchmark technology is measured from a specific (e.g. half-normal) distribution in which the parameters might differ. As pointed out by a referee, the LCSF model is similar to the stochastic frontier model with random coefficients introduced by Tsionas (2002), in the sense that a latent class model can be viewed as a discrete approximation to a (continuous) random coefficient model (see Greene, 2005, p.287).

Kumbhakar et al. (2013) have taken advantage of the latent class structure to introduce the so-called 'zero inefficiency stochastic frontier' (ZISF) model. This model overlooks the abovementioned issue of unobserved differences in technology or contextual factors, allowing the researcher to distinguish between fully efficient firms and firms that tend to be inefficient to some extent. In this sense, we hereafter state that the ZISF is able to deal with unobserved behavioural heterogeneity, i.e. hidden differences in firms' performance. The ZISF model is appealing in a benchmarking context, as it helps regulators to identify the utilities that can be used as "reference networks" for other (comparable) utilities. ${ }^{3}$ The present paper uses the ZISF approach to determine this reference network, extending the latter to also take into account the technological heterogeneity as well as geographical and weather conditions among networks.

Kumbhakar et al. (2013) assume there are only two types of firms (efficient and inefficient). While the inefficiency distribution for fully efficient firms is a point mass at 0 , the degree of inefficiency for inefficient firms is captured by any of the array of standard one-sided distributions, such as half-normal, exponential, or truncated normal. However, they use the latent class structure to identify unobserved differences in performance, assuming the estimated technology to be identical for all firms. Consequently, they abstract from technological heterogeneity among the firms and focus exclusively on the distribution of inefficiency.

Finite mixture models have traditionally been used to identify groups of firms that operate with different operating conditions or use different technologies. The issue is that the presence of one technology or another is not directly observed by the researcher. At most, only partial technological or environmental indicators are available. If the underlying data generation process only involves two technologies and there are only two types of firms (efficient and inefficient), we could then estimate a latent class model with four classes in which both technological and efficiency parameters differ. As both sources of unobserved heterogeneity (behavioural and technological) are treated symmetrically in such models, it becomes difficult to ascertain whether the differences in performance are caused by differences in behaviour or technology, and vice versa. In addition, it proves impossible to distinguish between the probabilities of sharing the same technology (i.e. being comparable firms) and the probabilities of sharing similar performances (i.e. being fully efficient or inefficient). ${ }^{4}$

The difference in the nature of behavioural and technological differences is not a semantic point. We utilize the difference in both of these sources of unobserved heterogeneity to develop a nested latent class model (hereafter NLC model), where the behavioural differences are modelled using two ZISF models. These are in turn nested into a latent class structure in order to capture the unobserved differences in technological or environmental conditions. See Figure 1 for a description

3. Several South American countries (e.g., Argentina, Chile, and Peru) use a rather similar concept, called "Model Company", to determine the allowed revenues, or allowed prices, of distribution companies (see Cossent, 2013). This approach relies on "building" engineering bottom-up models of a network company as benchmarking reference for a set of real firms, which is characterized in terms of network assets and associated costs, overhead structure and commercial costs, and the degree of population density (urban vs. rural) of the sectors or areas operated by each firm.

4. Kumbhakar et al. (2013, p. 67) state that "it is not clear from the finite mixture approach whether identifying a group of efficient firms is actually predicated on overfitting from allowing technological heterogeneity across the regimes". 
Figure 1: Structure of the Proposed Nested Latent Class Model

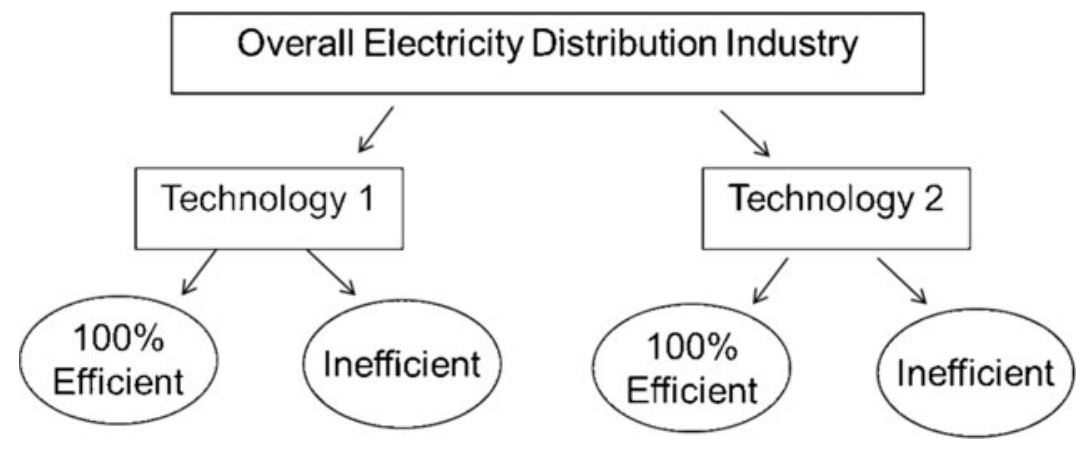

of the proposed model. To our knowledge, the present study is the first to propose a 'nested latent class' model. Hence, we extend the ZISF methodology in Kumbhakar et al. (2013) and provide a framework to distinguish between fully efficient and inefficient firms in a sample when the underlying technology is heterogeneous.

The paper is organized as follows. Section 2 introduces the LCSF and ZISF models and the econometric specification of our NLC model. In Section 3 we perform a Monte Carlo simulation to examine the relative performance of our NLC model in a controlled experiment. The data used in the empirical analysis is presented in Section 4. Section 5 presents and analyses the results obtained. Several policy implications are outlined and discussed in Section 6. Finally, Section 7 offers a summary and conclusions.

\section{METHODOLOGY AND MODELLING APPROACH}

This section develops a nested latent class model where unobserved differences in performance are modelled using two ZISF models nested in a LCSF model that, in turn, aims to capture unobserved differences attributable to technological or environmental conditions.

Let us first assume that there are $J$ different technologies, and that each firm belongs to one, and only one, of these technologies. Next, we adapt in a panel data setting the ZISF model introduced by Kumbhakar et al. (2013) to identify behavioural differences among electricity distribution firms. The model assumes that some firms are fully efficient, while others tend to be inefficient to some extent.

Conditional on technology $j(=1, \ldots, J)$, the general specification of the ZISF model can be written as follows:

$$
\ln y_{i t}=f\left(x_{i t}\right)+v_{i t \mid j}+u_{i t \mid j}
$$

where $i$ stands for firms, $t$ for time, $y_{i t}$ is a measure of firms' cost or other performance, $x_{i t}$ is a vector of cost drivers, $v_{i t \mid j}$ is a noise term that follows a normal distribution, and $u_{i t \mid j}$ is a one-sided error term capturing firms' inefficiency. While in the "inefficient" class we assume that $u_{i t \mid j} \sim N^{+}\left(0, \sigma_{u}^{2}\right),{ }^{5}$ the "fully efficient" class is defined by imposing that the variance of the pre-

5. For notational ease we here assume homoscedastic distributions for the inefficiency term. As this assumption could bias our parameter estimates, we use heteroscedastic specifications of $\sigma_{\mathrm{u}}$ in our empirical application. 
truncated normal distribution is zero, i.e. $\sigma_{u}^{2}=0 .{ }^{6}$ In other words, while the relevant model for the inefficient firms is the traditional stochastic frontier model with two random terms (SF model), the relevant model for the fully efficient firms is the neoclassical cost model that does not include any inefficiency terms (ZI model).

Given that the researcher lacks knowledge as to whether a particular firm is $100 \%$ efficient or not, the probability of being fully efficient or inefficient should be estimated simultaneously alongside other parameters of the model. Let us denote the probability of being inefficient as $\prod_{i \mid j}$. As we are interested in identifying firms that have persistently been fully efficient during the sample period, we do not allow $\prod_{i \mid j}$ to vary over time. ${ }^{7}$ Following Greene (2005) we parameterize the probability of being inefficient as a multinomial logit function:

$$
\prod_{i \mid j}\left(\gamma_{j}\right)=\frac{\exp \left(\gamma_{j}^{\prime} z_{i}\right)}{1+\exp \left(\gamma_{j}^{\prime} z_{i}\right)}
$$

where $z_{\mathrm{i}}$ is a vector of firm-specific variables which influence whether a firm is inefficient or not. The contribution of firm $i$ to the conditional (on technology-class $j$ ) likelihood is:

$$
L F_{i \mid j}\left(\theta_{j}\right)=L F_{i \mid j}^{S F} \cdot \prod_{i \mid j}\left(\gamma_{j}\right)+L F_{i \mid j}^{Z I}\left[1-\prod_{i \mid j}\left(\gamma_{j}\right)\right]
$$

where $\theta_{j}$ encompasses all parameters associated with technology class $j . L F_{i \mid j}^{S F}$ is the likelihood function of a SF model with two random terms, which is the applicable likelihood function when firms are inefficient, and $L F_{i \mid j}^{Z I}$ is the likelihood function of a normal random variable, which is the proper function when firms are fully efficient. Following Greene (2005, eq. 35), we model these two likelihood functions as follows:

$$
\begin{aligned}
& L F_{i \mid j}^{S F}=\prod_{t=1}^{T} L F_{i t \mid j}^{S F} \\
& L F_{i \mid j}^{Z I}=\prod_{t=1}^{T} L F_{i t \mid j}^{Z I}
\end{aligned}
$$

We next use the latent class structure to identify differences in technology among electricity distribution firms. The unconditional likelihood for firm $i$ is obtained as the weighted sum of their technology-specific likelihood functions, where now the weights are probabilities of technologyclass membership, $P_{i j}$. That is:

$$
L F_{i}(\theta, \delta)=\sum_{j=1}^{J} L F_{i \mid j}\left(\theta_{j}\right) P_{i j}\left(\delta_{j}\right)
$$

where $\theta=\left(\theta_{1}, \ldots, \theta_{J}\right), \delta=\left(\delta_{l}, \ldots, \delta_{J}\right)$, and the technology-class probabilities are again parameterized as a multinomial logit model:

6. We thank William Greene for reminding us that the "efficient" class cannot be defined by simply imposing $u_{i t \mid j}=0$, since a continuous random variable with a positive variance does not take a value of zero.

7. Grassetti (2011) also extended the ZISF model to the panel setting. In line with the advice of a referee, we develop a pooled specification of our NLC model in Appendix A that does not impose time-invariant class probabilities. This specification could be interesting in empirical analyses where firms can change their technology over time. For instance, dairy farms could change from an extensive milk production system to an intensive system, and vice versa. 


$$
P_{i j}\left(\delta_{j}\right)=\frac{\exp \left(\delta_{j}^{\prime} q_{i}\right)}{1+\sum_{j=1}^{J-1} \exp \left(\delta_{j}^{\prime} q_{i}\right)}, \quad j=1, \ldots, J-1
$$

where $q_{\mathrm{i}}$ is a vector of firm-specific variables. The last probability is obtained residually taking into account that the sum of all probabilities should be equal to one. Therefore, the overall likelihood function resulting from (2) and (7) is a continuous function of the vectors of parameters $\theta$ and $\delta$, and can be written as:

$$
\ln L F(\theta, \delta)=\sum_{i=1}^{N} \ln L F_{i}(\theta, \delta)=\sum_{i=1}^{N} \ln \left\{\sum_{j=1}^{J} L F_{i \mid j}\left(\theta_{j}\right) P_{i j}\left(\delta_{j}\right)\right\}
$$

Maximizing the above maximum likelihood function gives asymptotically efficient estimates of all parameters. The estimated parameters can then be used to compute (unconditional) posterior class membership probabilities for each technology, and (conditional) posterior class membership probabilities for both efficient and inefficient firms. The unconditional posterior probabilities can first be used to allocate each firm to a technology-class with highest probability, and then allocate the firm to a fully-efficient or inefficient class conditional to the technology-class allocation.

If $J=2$, our NLC model can be viewed as a particular case of a standard LCSF model with 4 classes and 4 class-membership probabilities. However, unlike a standard LCSF model, the likelihood function in (8) involves two different LFs. In order to capture an idea of the differences between the two specifications, we next assume that all variables are scalars. In this case, if we ignore any time and firm subscript, we can rewrite our technological probabilities $(\mathrm{P})$ and the behavioural probabilities ( $\Pi)$ as follows:

$$
\begin{array}{ll}
P_{1}=\frac{e^{\delta q}}{1+e^{\delta q}} & P_{2}=\frac{1}{1+e^{\delta q}} \\
\prod_{S F}=\frac{e^{\gamma z}}{1+e^{\gamma z}} & \prod_{Z I}=\frac{1}{1+e^{\gamma z}}
\end{array}
$$

By cross-multiplying the above probabilities we obtain the 4 final probabilities of our NLC model:

$$
\begin{aligned}
& P_{1}^{S F}=\frac{e^{\delta q+\gamma z}}{1+e^{\delta q}+e^{\gamma z}+e^{\delta q+\gamma z}} \\
& P_{2}^{S F}=\frac{e^{\gamma z}}{1+e^{\delta q}+e^{\gamma z}+e^{\delta q+\gamma z}} \\
& P_{1}^{Z I}=\frac{e^{\delta q}}{1+e^{\delta q}+e^{\gamma z}+e^{\delta q+\gamma z}} \\
& P_{2}^{Z I}=\frac{1}{1+e^{\delta q}+e^{\gamma z}+e^{\delta q+\gamma z}}
\end{aligned}
$$

The above mathematical expressions clearly show that our NLC model imposes a special structure upon the class-probabilities in a standard 4-class latent model. In particular, the nested 
nature of our model constrains the ratio of the two final probabilities to only rely on a subset of the estimated class-parameters and variables. For instance, $P_{1}^{S F} / P_{1}^{Z I}=e^{\gamma_{z}}$, and $P_{1}^{S F} / P_{2}^{S F}=e^{\delta q}$.

As pointed out by a referee, equations $(11 \mathrm{a}-11 \mathrm{~d})$ could lead us to deduce that some identification issues might appear if $q$ and $z$ have common elements. This issue is better discussed if we assume that $q=z$. In this case, the above equations collapse to the following equations:

$$
\begin{aligned}
& P_{1}^{S F}=\frac{e^{\tau z}}{1+e^{\delta z}+e^{\gamma z}+e^{\tau z}} \\
& P_{2}^{S F}=\frac{e^{\gamma z}}{1+e^{\delta z}+e^{\gamma z}+e^{\tau z}} \\
& P_{1}^{Z I}=\frac{e^{\delta z}}{1+e^{\delta z}+e^{\gamma z}+e^{\tau z}} \\
& P_{2}^{Z I}=\frac{1}{1+e^{\delta z}+e^{\gamma z}+e^{\tau z}}
\end{aligned}
$$

where $\tau=\delta+\gamma$. As in a traditional latent class model, our final class probabilities depend on a single set of variables with different coefficients. The unique difference is that the last coefficient is the sum of the previous ones. This restriction is automatically imposed once we nest the ZISF models into a (another) latent class structure. This makes our model slightly different as compared to more traditional latent class models where the coefficients of the prior class probabilities vary freely across classes.

One might be led to conclude from the foregoing discussion that our NLC model can be estimated by simply imposing the above-mentioned restrictions on the prior-probabilities, followed by an estimate of a traditional LCSF model. However, this would not be the case for two reasons. First, we must also impose that the frontier parameters of the two pairs of classes are the same. ${ }^{8}$ Second, the key difference between our NLC model and a four-class LCSF model is that the likelihood (density) function appended to each class varies in our NLC model. In order to better elucidate this feature, let us write the overall likelihood function resulting from (11a-11d) as:

$$
L F=\left(L F^{S F} \cdot e^{\gamma_{z}}+L F^{Z I} \cdot e^{\delta q}+L F^{S F} \cdot e^{\delta q+\gamma_{z}}+L F^{Z I}\right) /\left(1+e^{\gamma_{z}}+e^{\delta q}+e^{\delta q+\gamma_{z}}\right)
$$

Equation (13) shows that in our NLC model the likelihood function of a given class depends on whether we assume that all firms in that class are fully efficient or tend to be inefficient. In contrast, the likelihood function in a standard latent class model is the same for all classes, i.e. $L F^{S F}$ or $L F^{Z I}$ are used in all cases. Thus, our model cannot be viewed as a nested model in a statistical context. As Figure 1 shows, from a structural point of view, our model is only nested in a four-class LCSF model.

The above-mentioned feature of our model helps to attenuate some identification issues. Indeed, the coefficients of both $q$ and $z$ variables are easier to identify because they are multiplying two different likelihood functions ( $L F^{Z I}$ and $L F^{S F}$, respectively) when they appear alone in a prob- 
ability class. Moreover, we use the same $q$ and $z$ variables (i.e. $q=z=1$ ) in the simulation exercise in Section 3, and we do not find any problems in distinguishing the probabilities of being inefficient from the technological probabilities.

However, an additional identification problem must be discussed. Traditional latent class models suffer from the label- switching problems (see, e.g., Stephens, 2000). In our case, we have just pointed out that both $\delta$ and $\gamma$ can be estimated separately. However, we still need to decide whether they belong to the probability of being inefficient or the technological probabilities. This issue appears specifically when the (conditional) likelihood functions belong to the same parametric family. This is not the case in our model as both coefficients are attached to two different likelihood functions when they appear alone in a probability class. This characteristic guarantees the absence of pure label- switching.

\section{SIMULATION EXERCISE}

In this section we perform a Monte Carlo simulation to examine the relative performance of four competing models (i.e. basic SFA, ZISF, LCSF and NLC) in a controlled experiment. Our simulation exercise may be viewed as an extension to those carried out by Kumbhakar et al. (2013) and Rho and Schmidt (2015). In both papers, the frontier does not vary across the two classes of firms; only the existence or non-existence of inefficiency differs. Our simulation examines some of their issues, but under the existence of unobserved differences in technology. Our simulation should naturally be focused on whether the proposed NLC model distinguishes in practice between behavioural and technological classes, an issue that has obviously not been addressed in the abovementioned papers. In order to ease the understanding and reading of this section we avoid most of the technical details and results. The main features of our simulation exercises can however be found in Appendix B.

We consider two scenarios in our simulation. In S-type scenarios, we allow for small differences in technology in the sense that only differences in the slope parameter are allowed. In L-type scenarios, we allow for large differences in technology in the sense that both the intercept and the slope parameters differ. While the differences in the slope parameter are aimed at measuring differences in firm' scale economies (one of the main technological characteristics in electricity distribution), the differences in the intercept are focused on the measurement of the average effect of unobserved differences in weather or geographic conditions, demand features, etc.

Two key tuning parameters of our simulations are the ratio of inefficiency and noise standard errors, $\lambda=\sigma_{\mathrm{u}} / \sigma_{\mathrm{v}}$, and the proportion of inefficient firms (hereafter P). We first assume that $\mathrm{P}=0.8$. This value matches with the general view of many regulators of electricity distribution industries who consider that about $20 \%$ of regulated firms are efficient. This assumption is in line with efficiency improvement and evidence from Norway indicated a rather high average efficiency across the networks (see, e.g., Miguéis et al., 2012). For our model, we consider two possible $\lambda$ values. When $\lambda=1$, the inefficiency level of the inefficient firms is not very large since both the noise and the inefficiency term are of a similar magnitude. When $\lambda=2$, the differences in behaviour between inefficiency and fully efficient firms are larger. Therefore, the identification of fully efficient firms is easier in this case. Another source of identification problems is the proportion of inefficient firms (see Kumbhakar et al., 2013; and Rho and Schmidt, 2015 for an exhaustive analysis of this issue). In order to examine this issue in our framework with unobserved differences in technology, we simply reduce $\mathrm{P}$ in the latter model and set it equal to 0.6 .

In summary, our simulation exercise involves three alternative scenarios with small differences in technology and a similar number with large technological differences. In each scenario, 
Table 1: Correlation Between Simulated and Estimated Efficiency Scores

\begin{tabular}{ccccccc}
\hline Scenario & $\lambda$ & $\mathrm{P}$ & Basic SFA & ZISF & LCSF & NLC \\
\hline S1 & 1 & 0.8 & 0.425 & 0.375 & 0.409 & 0.470 \\
S2 & 2 & 0.8 & 0.624 & 0.640 & 0.744 & 0.782 \\
S3 & 2 & 0.6 & 0.620 & 0.681 & 0.739 & 0.817 \\
\hline & & & & & & \\
Scenario & $\lambda$ & $\mathrm{P}$ & Basic SFA & ZISF & LCSF & NLC \\
\hline L1 & 1 & 0.8 & 0.357 & 0.230 & 0.397 & 0.456 \\
L2 & 2 & 0.8 & 0.519 & 0.375 & 0.748 & 0.785 \\
L3 & 2 & 0.6 & 0.509 & 0.446 & 0.743 & 0.819 \\
\hline
\end{tabular}

we have estimated four competing models: basic SFA, ZISF, LCSF and NLC. The basic SFA model ignores both the simulated differences associated with the existence and non-existence of inefficient firms, and the differences attributable to firms' technologies. These shortcomings are partially addressed in the next two models. While the ZISF model tries to deal with the underlying behavioural differences, the LCSF model is mainly designed to capture unobserved differences in technology. Our more comprehensive NLC model aims to deal with both issues simultaneously. All models have been estimated using maximum likelihood.

In Table 1 we present the average correlation between the simulated and estimated efficiency scores. In Table 2 we provide the average correlation between the real (simulated) class memberships and the estimated sample allocations. All observations have been allocated to each technology-class and behavioural-class in accordance with the estimated posterior class probabilities.

Table 1 shows that, as expected, the basic SFA model performs poorly, particularly in Ltype scenarios when the ignored differences in technology are larger. The small correlation in the SFA model indicates that this model tends to over-estimate firms inefficiency (scores not shown) because we have assumed that all firms are inefficient to some extent, whereas in fact many of them are not. For this reason, the correlation between efficiency scores decreases a little when the proportion of inefficiency firms decreases to $60 \%$. Also, as expected, the correlation between efficiency scores is smaller when $\lambda=1$ and the underlying firms' inefficiency is rendered more important.

The performance of the ZISF model also worsens when the ignored differences in technology are larger. Interestingly enough, its performance in L-type scenarios is much poorer than the performance of a basic SFA model. That is, in contrast to Kumbhakar et al. (2013) that assumed a common technology in their simulation exercises, we do not find a better prediction of firms' efficiency when we control for fully efficient and inefficient firms. This seems to indicate that the ZISF model is highly sensitive to the existence of unobserved differences in technology, and as we anticipated in the introductory section of this paper, it may label incorrectly the unobserved differences in technology as being unobserved differences in efficiency.

The estimated coefficients in Appendix B indicate that the LCSF model is able to capture quite well the differences in both the intercept and the slope parameter. Consequently, the average correlations between the simulated and estimated efficiency scores in Table 1 using the LCSF model are much larger. This result thus seems to indicate that controlling for technological differences is much more important than controlling for unobserved differences in performance. The next set of results relating to the model's allocation power seems to confirm this conclusion. Our NLC model merits similar comments. Worthy of note is the fact that all figures are better when we move from the LCSF model to our NLC model. This in turn indicates that the LCSF model is still slightly biased because it ignores the co-existence of fully efficient and inefficient firms in the sample. 
Table 2: Correlation Between Simulated and Estimated Firms' Allocations

\begin{tabular}{|c|c|c|c|c|c|c|}
\hline \multirow[b]{2}{*}{ Scenario } & \multirow[b]{2}{*}{$\lambda$} & \multirow[b]{2}{*}{$\mathrm{P}$} & \multicolumn{2}{|c|}{ Behavioural allocation } & \multicolumn{2}{|c|}{ Technological Allocation } \\
\hline & & & ZISF & NLC & LCSF & NLC \\
\hline S1 & 1 & 0.8 & 0.304 & 0.403 & 0.857 & 0.860 \\
\hline S2 & 2 & 0.8 & 0.586 & 0.768 & 0.863 & 0.877 \\
\hline S3 & 2 & 0.6 & 0.665 & 0.822 & 0.867 & 0.892 \\
\hline Scenario & $\lambda$ & $\mathrm{P}$ & ZISF & NLC & LCSF & NLC \\
\hline L1 & 1 & 0.8 & 0.152 & 0.383 & 0.944 & 0.946 \\
\hline L2 & 2 & 0.8 & 0.241 & 0.762 & 0.939 & 0.950 \\
\hline L3 & 2 & 0.6 & 0.334 & 0.820 & 0.937 & 0.954 \\
\hline
\end{tabular}

In Table 2 we examine the allocation powers of each model using the computed correlations between real (simulated) class memberships and estimated class allocations. Scenario S3 for the ZISF model in this table indicates that the ZISF model tends to allocate firms "quite" well between the inefficient and fully efficient classes when the proportion of fully efficient firms is not too large and $\sigma_{u}$ is also relatively large compared to $\sigma_{v}$. Both Kumbhakar et al. (2013) and Rho and Schmidt (2015) obtained a similar result, but here we achieve it in a framework with unobserved differences in technology. In this sense, it should be highlighted that the correlation between the simulated and estimated class allocation in the ZISF model tends to deteriorate substantially when the differences in technology become much larger (i.e. when we move from S-type Scenarios to L-type Scenarios).

The figures for the LCSF model indicate that the allocation power of the LCSF model is very high, and improves when more differences in technology are allowed. It is also worth noting that its allocation power barely changes with (changes in) the level of the inefficient firms and with the proportion of fully efficient firms in the sample. On the other hand, the numbers for the NLC model in Table 2 suggest that the technological-class allocation power of our NLC model is even larger than in the LCSF model. The better technology-class allocation of our model in turn suggests that the technological probabilities are not (or at least are less) contaminated by the behavioural probabilities. To the contrary, the larger biases in technology-class allocation in the LCSF model seem to indicate that its technological probabilities are biased to some extent by the ignored behavioural differences in performance. A noteworthy result is the large improvement in behaviouralallocation power when we move from the ZISF model to our NLC model. Again as expected, the better performance is more significant when the differences in technology (ignored by the ZISF model) are larger. In general, our results show the poor performance of the ZISF in this framework.

To summarise, Table 2 helps us to discuss the identification issues of our NLC model, i.e. whether it is able to distinguish in practice between behavioural and technological classes. The large correlations that we find for the NLC model in both behavioural and technological-class allocations suggest overall that the proposed model does not suffer from serious identification problems, and that both types of classes are fairly well identified in our NLC model.

\section{DATA}

The data set used in this study is an unbalanced panel for Norwegian distribution utilities for the years 2004 to $2011 .{ }^{9}$ Norway presents a particularly suitable context and an interesting case

9. The data used in this study was obtained from the sector regulator, the Norwegian Water Resources and Power Directorate (NVE). The data for the period 2000-2003 is not used due to missing values in key variables, such as network size or cost of energy not supplied. Also, several firms were dropped due to lack of information on contextual variables or 
for which to implement the proposed methodology. First, Norway was among the first countries to introduce incentive-based regulation and efficiency benchmarking in 1997 (based on the DEA technique) in the electricity sector. Therefore, much of the managerial inefficiency of the networks has over time been removed. Second, Norway is the only country that explicitly incorporates quality of service in the form of the cost of non-delivered energy using estimated customer willingness-topay as an integrated part of the efficiency benchmarking exercise in incentive regulation of distribution networks. Third, unlike most countries, the Norwegian electricity sector consists of a large, though slowly declining (due to mergers and acquisitions), number of network utilities which allows the use of sophisticated analytical methods. ${ }^{10}$ Finally, the energy regulator has systematically examined the effects of environmental factors such as geographic and weather conditions on cost and service quality performance of the utilities and has reflected these in the efficiency benchmarking models (see, e.g., Growitsch et al., 2012; Orea et al., 2015). In particular, the regulator has analysed (selected) a large (small) number of geographic and weather variables that might affect the firms' cost function.

We specify a simple cost model that, pursuant to the Norwegian benchmarking approach, uses a measure of social costs (SCOST) as dependent variable that internalizes specific external costs to users in private production costs of the firms. In addition to operating expenses (OPEX), capital depreciation and opportunity cost of capital, the social cost dependent variable also includes the external costs of quality of service-i.e. cost of energy not supplied (CENS) to users due to service interruptions. CENS is calculated by multiplying the energy not supplied (KWh) during a specific interruption with a unit cost $(\mathrm{NOK} / \mathrm{KWh})$ that depends on customer type, duration, and whether the interruption was planned or not. ${ }^{11}$ Finally, OPEX also includes the cost of network energy losses obtained by multiplying the units of network energy losses with the average system price in NordPool wholesale market in a given year. The monetary variables finally used in our application are measured in $1000 \mathrm{NOK}$ and have been deflated using the consumer price index to express them in 2004 real terms.

Regarding the cost drivers, our cost frontier includes three outputs (CUS $=$ number of customers; $\mathrm{NL}=$ network length, $\mathrm{Km} ; \mathrm{DE}=$ delivered energy ${ }^{12}, \mathrm{MWh}$ ), and three input prices ( $\mathrm{PK}$ = capital price, regulated return of capital; $\mathrm{PE}$ = energy price, system price in NordPool Spot; $\mathrm{PL}=$ labour price, a wage dominated index). In addition, we include selected environmental variables in our analysis that are considered as relevant by the Norwegian regulator. We include two weather variables: WIND = average arithmetic reference wind from measuring stations $\mathrm{m} / \mathrm{s}$ and WINDEX = average wind exposure based on 25-30 years observation, $\mathrm{m} / \mathrm{s}$. We also use a geographic variable (DIS = average arithmetic distance of some reference points to coast, $\mathrm{Km}$ ) in order to capture the effect of coastal climate on the networks. In Norway, this effect is related to problems with corrosion on network components normally caused by a combination of wind and salt water. ${ }^{13}$

because they had unreasonable data, e.g. OPEX or CENS equal to zero, or negative values for new investments. Although our panel data is not balanced, most of the firms are observed during the whole sample period. The number of firms (119 on average) varies slightly over time due to a reduced number of mergers.

10. Following a merger, NVE only collects and reports data for the merged entity.

11. We are grateful to a referee for pointing out the full definition of CENS in Norway.

12. Please note that since 2013, the Norwegian regulator no longer uses DE as an output and instead uses number of customers, length of network, and number of network stations as outputs (see Amundsveen and Kvile, 2015).

13. The regulator uses the ratio of squared wind speed over distance to coast in order to reflect the combined effect of these variables. NVE also uses a measure of forest density in the service areas of networks. Moreover, the regulator considers a range of variables in pre-benchmarking analysis to account for the effect of different degrees of forestation in the service area. We encountered convergence problems when we included the whole set of variables that are available in our data set 
As we use the logarithmic measure of this variable, for a given increase in distance, this will have a higher percentage effect on the cost of firms located closer to the coast. We use the percentage of overhead lines $(\mathrm{OH})$ of total network length in $\mathrm{Km}$ as an additional cost driver. This variable is employed to represent the main technical feature in this industry as firms' decisions on, for example, investment and maintenance for overhead and underground lines are different. It should be noted that the $\mathrm{OH}$ variable is also used in other parts of the model in order to capture not only differences in technology, but also in firms' performance. We expect the effect of environmental conditions on firms' costs to depend on the technological characteristics of their networks. In particular, overhead lines are significantly less costly than underground cables but they also tend to have less reliability due to their exposure to weather factors. Therefore, we also interact the percentage of overhead lines with WIND, WINDEX and DIS.

The technological-class probabilities are also functions of $\mathrm{OH}$ in order to test whether other and unobserved technological differences are related to the percentage of overhead lines. We associate a low share of overhead lines in total network length with more urban service areas. ${ }^{14}$ These service areas tend to have higher customer density which makes it cost efficient to use underground cables which in turn have higher reliability lower CENS. In principal, it is possible to specify more elaborate models to capture technological heterogeneity but these will vary from country to country. We estimated some more elaborate models but these produced similar results.

Regarding firms' inefficiency, we use the percentage of overhead lines and network length variables (i.e. $\mathrm{OH}$ and NL) and the number of stations (ST) either as inefficiency determinants or determinants of being inefficient. Finally, a time trend is included as a determinant of firms' inefficiency to check whether the Norwegian regulation has been successful in improving the efficiency of the firms.

Table 3 provides a descriptive summary of the variables used in this study.

\section{RESULTS}

\subsection{Parameter Estimates}

We estimate four alternative model specifications for our empirical analysis. Table 4 shows the estimated coefficients of the cost models. The RSCFG model assumes that the inefficiency term follows a heteroscedastic half-normal distribution. ${ }^{15,16}$ This empirical strategy not only allows us to obtain consistent estimates of both frontier coefficients and firm-specific inefficiency scores, but

in order to account for forest conditions. Using an aggregate measure, the convergence problems disappeared. The efficiency scores were not only highly correlated in all estimated models, but also the identification of fully efficient firms in the NLC model is almost the same regardless of whether we control for the differences in forest conditions. Therefore, this result strongly corroborates the use of our NLC model when data for some relevant cost drivers are not available or are costly to collect, which is often the case in regulatory settings.

14. We could instead use some measure of customer density to distinguish between urban and rural networks. However, in terms of the effects on firm technology, the effect of customer density on network technology works through its effect on technical features of networks such as the share of overhead vs. underground cables.

15. This model is labelled as RSCFG as it was introduced by Reifschneider and Stevenson (1991), Caudill and Ford (1993) and Caudill, Ford and Gropper (1995). The efficiency covariates in these papers are treated as determinants of the variance of inefficiency term.

16. The efficiency covariates in this model are treated as determinants of the variance of inefficiency term. As pointed out by Alvarez et al. (2006), this specification satisfies the so-called scaling property, which has several appealing features, and tends to yield less convergence problems than other heteroscedastic specifications of the SFA model. 
Table 3: Descriptive Statistics of the Data

\begin{tabular}{c|cccc}
\hline & Mean & St.Dev. & Min & Max \\
\hline SCOST & 77700.02 & 132024.01 & 2343.05 & 793884.71 \\
PK & 0.06 & 0.01 & 0.05 & 0.08 \\
PL & 163.67 & 16.99 & 139 & 189.5 \\
PE & 331.02 & 73.94 & 234.6 & 436.3 \\
CUS & 16753.17 & 33229.86 & 348 & 182746 \\
ST & 809.9 & 1381.62 & 29 & 9428 \\
DE & 432406.39 & 875129.47 & 6915 & 5200000 \\
NL & 661.83 & 1036.34 & 30 & 6542 \\
WIND & 25.5 & 2.44 & 22 & 31 \\
WINDEX & 5.28 & 1.04 & 2.71 & 8.13 \\
DISTANCE & 53824.79 & 55649.33 & 190.96 & 196377 \\
OH & 0.68 & 0.19 & 0.14 & 0.97 \\
\hline
\end{tabular}

also to incorporate determinants of firms' inefficiency. The ZISF model is a panel-data and heteroscedastic version of the (homoscedastic) model introduced by Kumbhakar et al. (2013). Unlike these authors, the inefficiency term here is again specified to be firm-specific. The ZISF model only captures unobserved differences in firm performance as it disregards the presence of unobserved differences in technology. In contrast, the LCSF model allows us to control for unobserved technological differences among firms. As it does not distinguish between fully efficient and inefficient utilities, we state hereafter that the LCSF model does not control for unobserved behavioural differences among firms. Like the ZISF model, the heteroscedastic specification of the LCSF model allows for behavioural differences. However, while these differences in the LCSF model are only related to the magnitude (shape) of the inefficiency term, the behavioural differences captured in the ZISF model pose a stronger dilemma: the existence or absence of inefficiency. Finally, the NLC model takes into account both behavioural and technological differences among firms using the specification outlined in Section 2.

Our four models are estimated using a (restricted) translog cost function that can be interpreted as a second-order approximation of the companies' underlying cost function. We therefore add the input prices to our cost function because they do not vary across utilities, but vary over time. This precludes using quadratic terms and interactions with these variables. A time trend is not included in the cost frontier also because it is highly correlated (99\%) with the labour price. As usual, homogeneity of degree one in prices is imposed by normalizing cost, labour price and capital price with the energy price. Each explanatory variable is measured in deviations with respect to its mean, such that the first-order coefficients in Table 4 can be interpreted as the cost elasticities/ derivatives evaluated at the sample mean.

In both LCSF and NLC models we assume the existence of only two classes to focus our discussion on the particular characteristics of the proposed model. We found in Llorca et al. (2015) and others papers estimating latent class models that a reasonable and practical trade-off between a good description of the data and sheer complexity is quite often provided by a model with two classes. The number of classes in a latent class framework could be examined using several information criteria tests (see, e.g., Orea and Kumbhakar, 2004). Even if two classes had not been the case in our application, we would still have expected our efficiency scores to be only slightly underestimated because the largest change in efficiencies is likely to occur when we move from one class to two classes (see Llorca et al., 2014). 


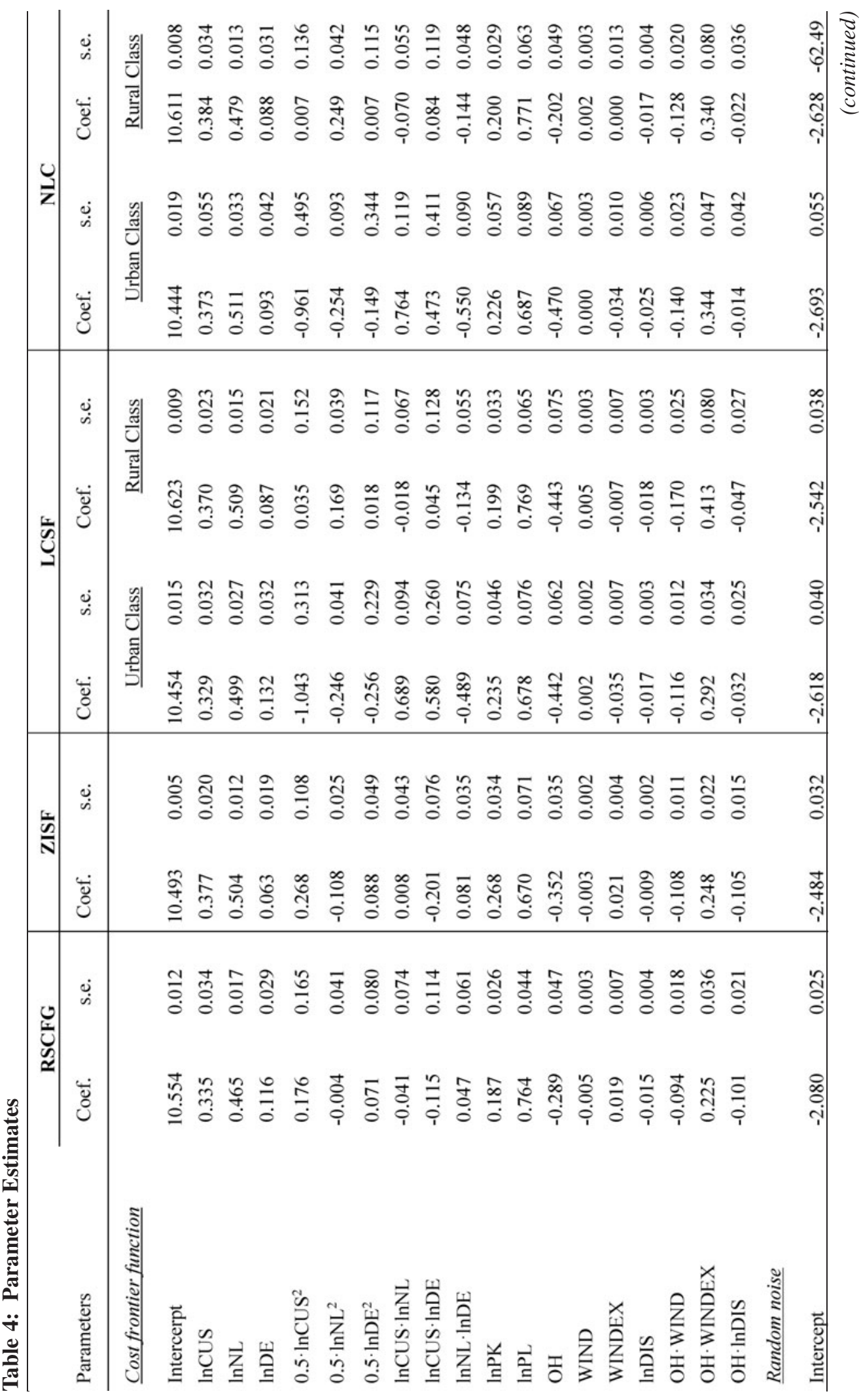

Copyright (C) 2017 by the IAEE. All rights reserved. 


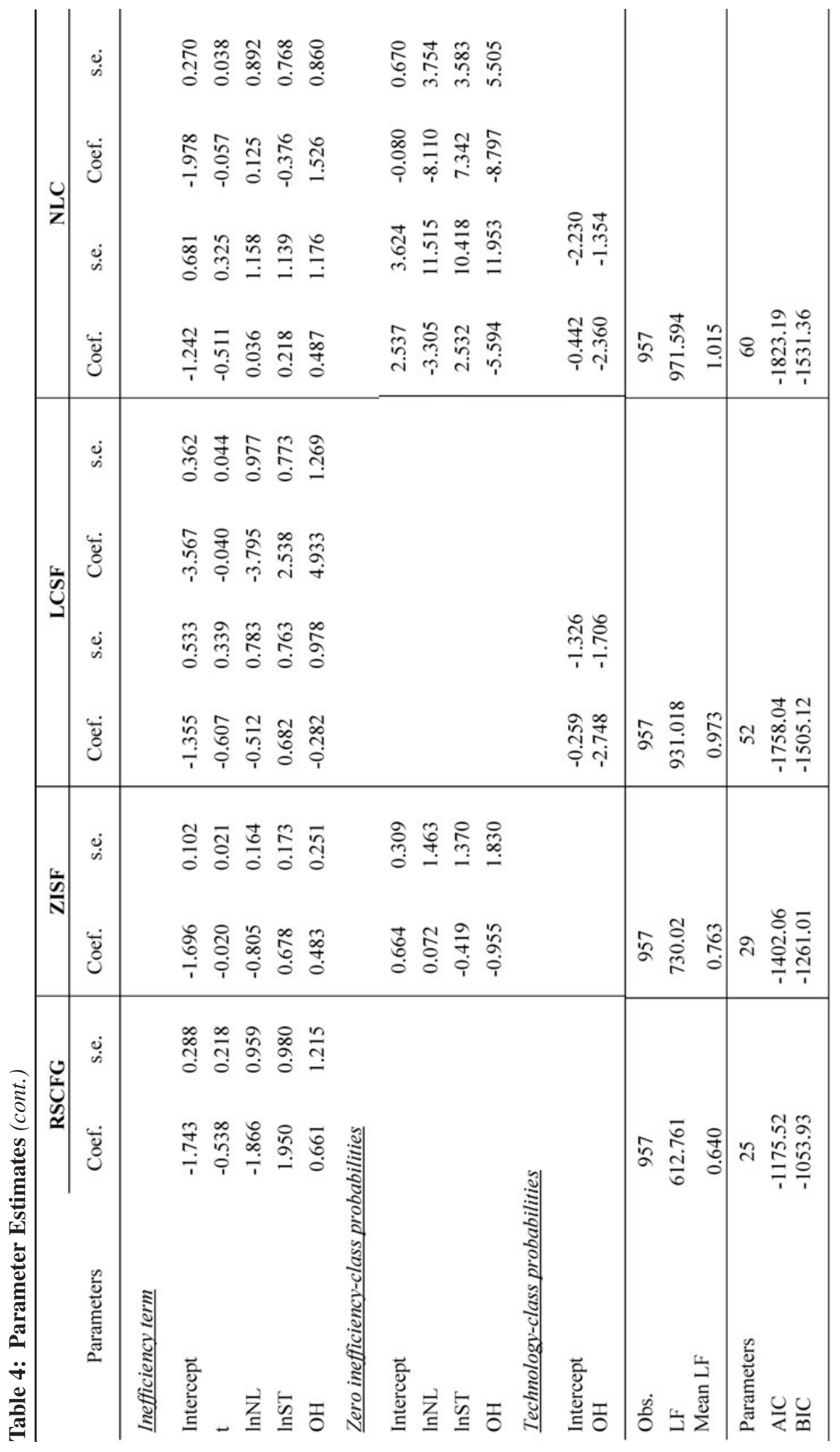

Copyright (C) 2017 by the IAEE. All rights reserved. 
As our results might depend on the empirical strategy chosen, in order to allow for unobserved differences in both technology and firms' performance, it is worth examining the goodness-of-fit of the four alternative specifications of our cost model. Given the estimated values of the likelihood function in Table 4, we can conclude that the well-known AIC and BIC tests would allow us to reject the RSCFG model in favour of the ZISF model. This implies that, in our application, it is important to control for unobserved differences in firms inefficiency. Therefore, ignoring the presence of the two types of firms (fully efficient vs. inefficient) in our sample, might bias both efficiency scores and technological coefficients. The RSCFG model would also be rejected in favour of the LCSF model, indicating that controlling for unobserved differences in firm technology (and in the determinants of firm inefficiency) is also important in our application. The previous three specifications would, in turn, be rejected in favour of the NLC model that allows us to include both types of unobserved heterogeneity. Based on these comparisons, we conclude that the NLC model is preferred, and that the results for firm technology and efficiency using the more restrictive RSCFG, LCSF and ZISF models should be interpreted with some caution.

In general, all models perform quite well as all of the first-order coefficients have the expected sign and their magnitudes are also reasonable from a theoretical point of view. The firstorder coefficients of all three outputs are positive and statistically different from zero. A similar observation can be made with respect to the coefficients of input prices, which are also positive and statistically significant. The sum of the first-order coefficients of customer numbers and energy delivered, allows us to measure density economies (i.e. output expansion in the existing service areas). The estimated coefficients for these two outputs indicate that electricity distribution networks have natural monopoly characteristics when an additional network is not required to meet additional demand. ${ }^{17}$ Obviously, the returns to scale are less if meeting the extra demand requires enlarging the current network. The scale economies can be computed adding the coefficients of the three output variables. The estimated coefficient for network length suggests that the scale economies are about 10 percentage points less than the density economies.

The frontier coefficient of $\mathrm{OH}$ is negative and statistically significant in all models, indicating that the larger the percentage of overhead lines, the smaller is the total cost. This result indicates that, although underground cables tend to be negatively correlated with CENS (the correlation between CENS/DE and $\mathrm{OH}$ ratio is -0.29) and reduce OPEX, they are also more costly and increase the total costs. The LCSF and NLC models in turn indicate that the technology in this industry exhibits some heterogeneity. Although the output elasticities evaluated at the sample mean are similar in the two classes in both models, the role of technological heterogeneity is particularly appreciated when we compare the second-order coefficients of the three outputs.

The estimated coefficients for the weather variables (WIND and WINDEX) and the distance to coast geographic variable (DIS), suggest that there are notable differences among the utilities in those costs attributable to different environmental conditions. It is worth mentioning that most coefficients of $\mathrm{OH}$ interacting with these three contextual variables are statistically significant, indicating that the effect of any of the weather variables is larger when the importance of overhead lines increases. While the coefficients of WIND are negative (but rarely significant), the effect of WINDEX on firms' costs is mostly significant and positive indicating that a higher exposure to wind implies larger costs to the distribution networks. The combinations of both wind variables and the distance to coast with the $\mathrm{OH}$ variable indicates that inland weather conditions are, as

17. Also Salvanes and Tjøtta (1998) find evidence of natural monopoly characteristics in the Norwegian electricity distribution networks.

Copyright (C) 2017 by the IAEE. All rights reserved. 
expected by the regulator, likely to be less severe than coastal weather conditions. On the other hand, the coefficient of the distance to the coast itself is always negative.

We have performed several Wald tests to examine whether the environmental variables are jointly significant. We rejected that they are simultaneously equal to zero in all models. Interestingly, using the LCSF and NLC models, we could not reject that their coefficients are the same in both technological classes. This seems to indicate that the unobserved heterogeneity has mainly to do with the effect of economic-based cost drivers (i.e. customers, delivered energy, network length, etc.). On the other hand, and as suggested by a referee, we have also included the percentage of sea cables to control for the share of them given the fact that sea cables are by far the most expensive type of cables. The coefficient of this variable was not significant in our NLC model either as a cost driver or as a determinant of the technological probabilities, and the sample partition proved the same as before. Results using the LCSF model deserve similar comments and overall these outcomes seem to indicate that the latent structure of each of the LCSF and NLC models is already capturing the sea cables issue, conditional on the set of explanatory variables. Furthermore, the percentage of sea cables is correlated with the DIST and WIND and WINDEX variables.

In addition to the frontier parameters, Table 4 displays the coefficients of the variables that are related to the inefficiency term, as determinants of either the inefficiency term or the probability of being inefficient. Although the significance of inefficiency determinants varies between models and classes, several results common to the four alternative specifications of our cost model are worth mentioning.

First, the negative sign for the time trend also suggests, albeit not always significant, that the regulation system in Norway has incentivised firms to improve their performance during the sample period. Second, we obtain a negative coefficient for NL in both RSCFG and LCSF models indicating that larger utilities tend to be more efficient than smaller utilities. In contrast, the positive coefficients of ST and $\mathrm{OH}$ indicate that it is more costly to manage firms with more stations and with higher share of overhead lines. These can also be viewed as measures of complexity of networks something that regulatory benchmarking models are currently lacking. Third, none of the aforementioned technological characteristics have significant effects on the distribution of inefficiency in the so-called urban class of the LCSF model (the labels are justified in the next subsection). They are also not statistically significant when we move to the NLC model that distinguishes between inefficient and fully efficient firms. Hence, we do not find evidence that these technology features make the operation of these distribution networks more costly. Regarding the rural class, here none of the technological features considered have a significant effect on the distribution of inefficiency for the inefficient farms, but two of them (network length and number of stations) do have significant effects on the probability of being fully efficient. ${ }^{18}$ Therefore, as pointed out by a referee, the inclusion of behavioural and technological class features in the model is relevant to the significance of inefficiency determinants, as well as to the conclusions that can be derived from this analysis.

While the technological variables included as efficiency determinants are still significant in the ZISF model, the probability of being inefficient does not depend on any covariate. This indicates that each firm has the same probability of being fully efficient, and they cannot use their size or other characteristics of their network as a reason for not being $100 \%$ efficient. A similar comment can be made regarding one of the classes of the NLC model since the probability of being 
Table 5: Average Values for Each NLC Class

\begin{tabular}{c|cccc}
\hline \multirow{2}{*}{ Variable } & \multicolumn{2}{|c}{ Technological classes } & \multicolumn{2}{c}{ Behavioural classes } \\
\cline { 2 - 5 } & Urban & Rural & Efficient & Inefficient \\
\hline CUS & 23938 & 11959 & 13753 & 18737 \\
DE & 631074 & 299846 & 344410 & 490612 \\
ST & 1004 & 680 & 836 & 793 \\
NL & 816 & 559 & 742 & 609 \\
DENSITY & 24.5 & 19.3 & 15.6 & 25.2 \\
OH (\%) & $63 \%$ & $71 \%$ & $78 \%$ & $61 \%$ \\
\hline Observations & 383 & 574 & 381 & 576 \\
\hline
\end{tabular}

Note: DENSITY is measured as CUS/NL

inefficient in the urban class does not depend on any covariate. In contrast, the probability of being inefficient in the rural class decreases with network size and increases with number of stations.

Regarding the determinants of the technological probabilities, the coefficient of $\mathrm{OH}$ is negative, indicating that firms with a greater percentage of overhead lines tend to belong to the rural class. This is an expected outcome as firms with more overhead lines are more likely to be serving rural areas. Following advice from a referee, we have estimated more elaborated models including environmental variables (WIND, WINDEX and DIS) as determinants of the technological probabilities. They were not statistically significant and the sample partition was completely invariant to their inclusion. Similar comments can be made regarding the inclusion of a density measure, which was not significant because higher customer density in urban areas tends to be associated with underground cables (and hence negatively correlated with $\mathrm{OH}$ ).

\subsection{Sample Partitions}

Table 5 provides the average value of some of variables included in the empirical analysis for each technological and behavioural class. ${ }^{19}$ Though Norway is not a very populated country, the figures in Table 5 allow us to label the two technological classes respectively as (more) urban and rural. Indeed, the firms in the first class have more customers on average and deliver more electricity than the utilities included in the second class. As expected, the density in the urban class is much larger than in the rural class. In addition, the percentage of overhead lines in the urban class is relatively small (63\%) compared to the percentage of overhead lines in the rural class (71\%). The rural areas are often broader than the urban areas. This in turn explains the larger number of stations per kilometre of network in the rural class. Regarding the behavioural classes, and in accordance with the estimated coefficients in Table 4, we conclude from Table 5 that the inefficient firms are larger (in terms of number of customers and electricity delivered) than the fully efficient firms, and tend to be operating in more populated areas (i.e. with larger density) where the percentage of overhead lines is less than in a rural area.

In Table 6 we compare the sample partition of the NLC model that controls for unobserved differences in technology and firm behaviour with those obtained using LCSF and ZISF that only

19. To this end we have used our more comprehensive NLC model. Similar results are obtained using the LCSF model. We have dropped those variables where the mean differences were not statistically significant. 
Table 6: Sample Partitions

\begin{tabular}{cccccc}
\hline \multirow{2}{*}{ ZISF } & \multicolumn{2}{c}{ LCSF } & \multicolumn{2}{c}{ NLC } \\
\cline { 3 - 6 } & & Urban & Rural & Urban & Rural \\
\hline Inefficient & 623 & 422 & 535 & 327 & 249 \\
Efficient & 334 & - & - & 56 & 325 \\
All & 957 & 422 & 535 & 383 & 574 \\
\hline
\end{tabular}

capture differences in one of the above-mentioned dimensions. The last row in Table 6 indicates that most observations belong to the rural class regardless of the model. However, while our preferred model allocates $60 \%$ of the observations to the rural class, the LCSF model balances out this allocation slightly as the larger (smaller) class only includes $56 \%(44 \%)$ of the sample.

The first two rows in Table 6 are focused on the identification of fully efficient and inefficient firms using the ZISF and NLC models. These rows firstly indicate that 335 observations (i.e.42 firms) are identified as fully efficient firms in the ZISF model, which represents $35 \%$ of all observations in our sample. In contrast, $40 \%$ of the observations (i.e. 48 firms) are labelled as fully efficient firms in the NLC model. In other words, some firms are wrongly labelled as inefficient in the simple ZISF model because their inefficiency scores have been computed using common cost frontiers and common efficiency coefficients (two assumptions that are rejected in our application). In addition, 32 firms in the ZISF model are wrongly labelled as fully efficient as they are not identified as fully efficient using our preferred NLC model. Note as well that all firms in the LCSF model are inefficient to some extent, while only $43 \%$ of observations allocated to the rural class in the NLC model are labelled inefficient. This problem is less severe in the urban class as this percentage increases up to $85 \%$. Overall this explains why for the rural class we find a relatively small correlation (78\%) between the efficiency scores of the LCSF and NLC models.

For robustness analysis, we also estimate our models allowing the membership probabilities to vary across the two regulatory periods covered by our data set. ${ }^{20}$ Indeed, while our first three years (from 2004 to 2006) belong to the 2002-2006 price control review, the remaining years of our data set (from 2007 to 2011) cover the duration of the posterior price control review. ${ }^{21}$ When we relax the assumption of time-invariant class probabilities, the percentage of fully efficient firms in the ZISF model increases to 39\%. The results from the ZISF model with two sub-periods also indicate that fully-efficient behaviour is more frequent in the second sub-period (46\%) than in the first one (26\%). In the NLC model with two sub-periods, the percentage of fully efficient firms is fairly similar $(40 \%)$ to the percentage obtained in the case of complete time invariant class probabilities (39\%). As in the simple ZISF model, fully-efficient behaviour is more frequent in the second sub-period than in the first one.

Also for robustness purposes, we estimated more restrictive homoscedastic versions of our cost models, but the performed model selection tests rejected these specifications in favour of their heteroscedastic counterparts. ${ }^{22}$ Although these models provided very similar coefficients for the cost function, the number of fully efficient firms tends to increase when the inefficiency determinants and the determinants of inefficiency probability are not included in the models. By way of com-

20. The parameter estimates of these models are available from the authors upon request.

21. A new regulation regime started in 2007 but the firms did not have enough time to grasp the mechanisms and incentives of the new scheme in the first year. We are thankful to an anonymous referee for bringing this to our attention.

22. The homoscedastic estimates are available from the authors upon request. 


\section{Figure 2: Annual Efficiency Scores}

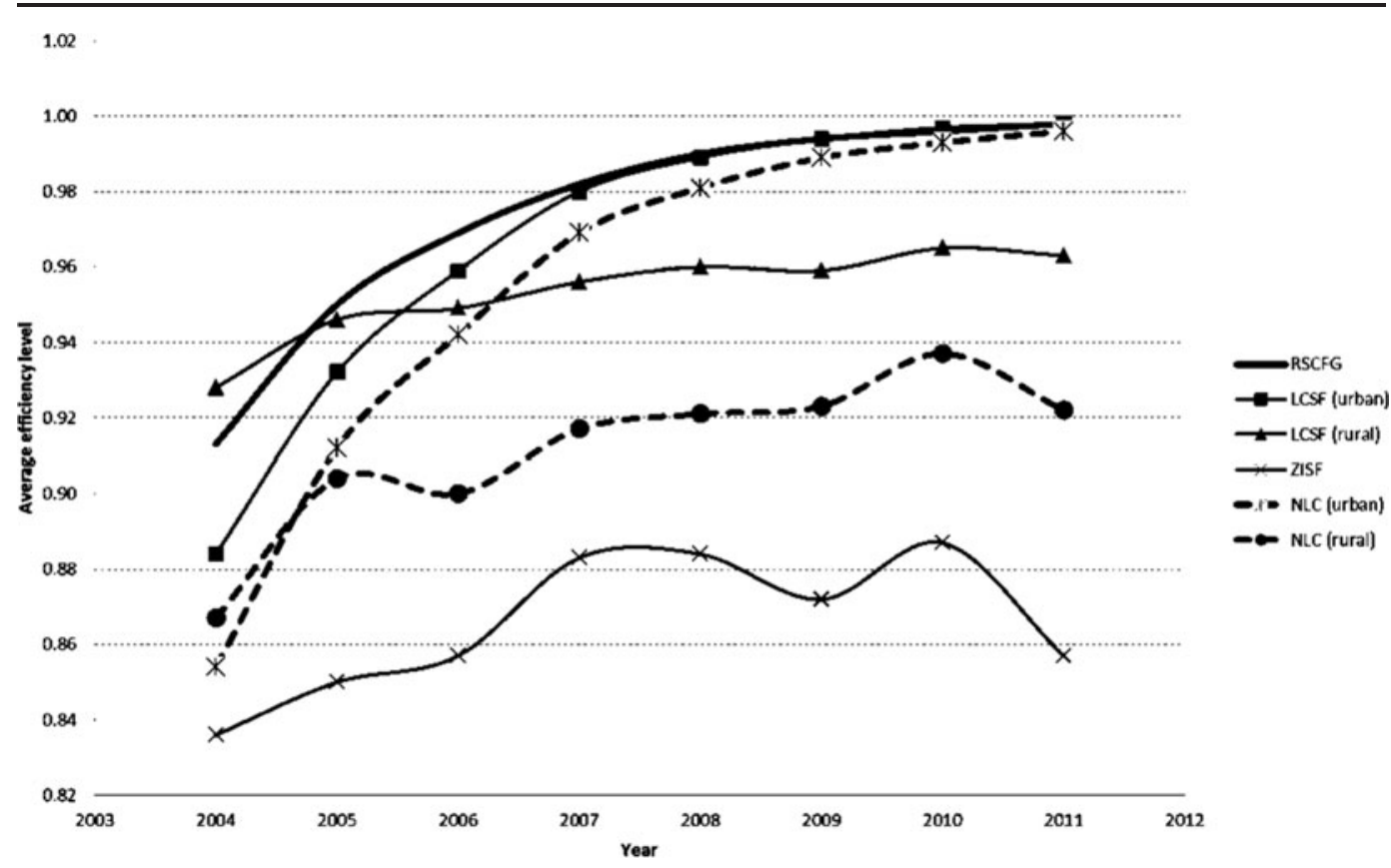

parison, we estimated the model using a two-stage procedure, even though a single-step approach is preferred. Once the LCSF model is estimated, we estimated a separate ZISF model for each class. The correlation coefficient between the efficiency scores is quite high (91.5\%) and the sample partitions are also relatively robust. However, some firms are wrongly labelled as inefficient and vice versa.

\subsection{Efficiency Scores}

Figure 2 depicts the annual average efficiency score of all firms in the case of the RSCFG and LCSF models. For the ZISF and NLC models, only the average efficiency score of those firms that are not fully efficient is presented. Our efficiency estimates are high, ranging from 87 to $97 \%$. The high level of efficiency of this industry is most probably attributable to the maturity of the Norway as a regulator that has consistently been supervising and incentivizing the Norwegian utilities to perform efficiently. Similar figures are obtained in Miguéis et al. (2012) using a DEA method for the period 2004 to 2007, and in Growitsch et al. (2012) using a SFA approach for the period 2001 to 2004 . The latter authors also found that efficiency estimates are strongly dependent on the empirical strategy used to control for observed and unobserved heterogeneity of the firms.

The average efficiency level of the inefficient firms in the ZISF model is $87 \%$. The estimated efficiency level of these firms in the RSCFG model is much higher, 97\%. This result thus tends to confirm Kumbhakar et al. (2013) findings, but with the variant now that we use heteroskedastic specifications of both SFA and ZISF models: the efficiency scores in a standard SFA model are overestimated if we are not able to identify the set of fully efficient firms. The same comment can be made regarding the inefficient firms of the rural class of the NLC model as their average efficiency level (91\%) is far from that estimated (96\%) using the RSCFG model. However, the 
average efficiency level of the inefficient firms in the urban class of the NLC model is not seriously over or under-estimated as the efficiency score using the RSCFG model is of similar magnitude on average.

It is interesting that the patterns of LCSF and NLC in the rural class are similar to the ZISF pattern, while the patterns of LCSF and NLC in the urban class are similar to the RSCFG pattern. Both ZISF and RSCFG ignore unobserved differences in technology, but provide a different treatment to the existence of fully efficient firms in the data. This result is reasonable because the percentage of fully efficient firms in the urban class is only $15 \%$. Hence, the pattern in the urban class tends to mimic the pattern of the model that ignores the existence of fully efficient firms. In contrast, the percentage of fully efficient firms in the rural class is quite high, about $57 \%$. Thus, the pattern in this case tends to mimic the pattern of the ZISF model that allows for fully efficient firms.

On the other hand, the computed coefficients of correlation between the NLC and the ZISF and LCSF efficiency scores indicate that ignoring unobserved differences either in technology or in firms' behaviour might seriously over or under-estimate the ranking of firms in accordance with their estimated efficiency levels. For instance, the computed coefficient of correlation between the ZISF and NLC efficiency scores is only about $42 \%$. This correlation drops to $25 \%$ if we only use the observations belonging to the urban class of this model. In the rural class, the correlation between LCSF and NLC models is relatively large, but far from $100 \%$.

\section{POLICY IMPLICATIONS}

In this section we summarize and discuss some of the policy implications that could be inferred from our paper. First of all, both our simulation exercise and our empirical application suggest that controlling for differences in technology is more important than controlling for differences in performance (in our case using a model that allows identifying fully efficient firms or modelling firms' inefficiency). In this sense, for instance, regulators benchmarking utilities should try to use some of the models proposed in the literature to control for unobserved environmental factors that might have an effect on regulated utilities' costs. In our case, we propose using a latent class model approach. Other options are obviously available. For instance, depending on their human resources, regulators may prefer using other parametric or non-parametric frontier techniques to achieve this aim (see, for instance, Fried et al, 2008). A complementary strategy is to collect all the relevant environmental data (i.e. the so-called contextual or non-controllable variables). Obviously, this strategy will again require a substantial amount of human or financial resources as well as time.

Our paper therefore advocates prioritizing actions aiming to better fit firms' technology over those actions aimed at the control of (un)observed differences in performance. Obviously, this is more relevant in countries (e.g. Germany, Sweden, Finland, Peru and Brazil) where electricity distribution networks are operating in regions with diverse weather and geographic conditions, population structure and settlement configurations, electricity demand patterns, density degrees (urban vs. rural), etc. Even though regulators in these countries are making a great effort to collect data to properly control for some of the aforementioned differences in environmental conditions, they still need to control for those unobserved (sometimes huge) differences which might jeopardize most of the benchmarking exercises carried out by the regulators.

The regulators may also be interested in identifying fully efficient network utilities that can be used as "reference networks" for other (comparable) utilities. The zero inefficiency stochastic frontier model introduced recently by Kumbhakar et al. (2013) can be used to achieve this aim. 
However, this model does not control for unobserved differences in technology or environmental conditions. In this sense, our results are useful for regulators (e.g. in Chile and Argentina) that use the so-called "model company" approach to identify reference networks to benchmark utilities (Jamasb and Söderberg, 2010; Silva, 2011). In these countries, engineering bottom-up models are built to provide an estimation of the efficient costs that would be incurred by a distribution company operating in a certain geographical area. The fully efficient firms identified in our NLC model can be viewed as "real-world" counterparts of some optimally designed grids that are built through engineering models. Our results are thus useful to examine the robustness of the engineering reference networks that ignore all the inner complexities of real networks, the potential trade-offs they face, the existence of both random shocks and unobserved differences in environmental conditions beyond the control of the distribution firms.

Finally, the fact that we have found $40 \%$ of fully efficient firms in the Norwegian electricity distribution industry, should be viewed as additional empirical evidence that backs the effort made by the NVE regulator to encourage Norwegian networks to perform better. That is, the great efficiency of this industry is as stated previously a direct consequence of the maturity of the Norwegian regulator that has been supervising and incentivizing the Norwegian utilities to perform efficiently. On the other hand, we should take into account that the above percentage of fully efficient firms may be slightly overestimated in our application due to the efficiency level of these firms which is traditionally very large (see, e.g., Miguéis et al., 2012, and Cheng et al., 2014). In this sense, Rho and Schmidt (2015) pointed out that the ZISF model (or extensions of this model such as that proposed in our present paper) might work well in other (less mature) electricity distribution industries all over the world.

\section{CONCLUSIONS}

In many countries, electricity regulators aim to measure the network utilities' efficiency against best practice performance. However, errors in identifying the correct benchmark firms or measuring their efficiency have important financial implications for all the less efficient firms against which they are compared. Therefore, obtaining reliable measures of firms' inefficiency often requires controlling for unobserved differences in the firms' technology or in the geographical and weather conditions under which each utility operates. Several well-known latent class stochastic frontier models now allow researchers (and regulators) to account for the above-mentioned technology heterogeneity.

The present paper extends the ZISF approach to take into account the heterogeneity in firms' technology as well as in their environmental conditions. We take advantage of the differences in the nature of both sources of unobserved heterogeneity to develop a nested latent class (NLC) model. The behavioural differences are modelled using two ZISF models that are in turn nested into a latent class structure that aims to capture unobserved differences in technology or environmental conditions. To our knowledge, the present study is the first to propose a nested latent class model to distinguish between fully efficient and inefficient firms when the underlying technology is heterogeneous. The present paper is also the first to introduce the zero-inefficiency approach in a regulatory context.

We illustrate the proposed models with an application to the Norwegian distribution network utilities for the period 2004-2011. Following the Norwegian benchmarking approach, four alternative specifications of a cost model are estimated. Overall our results suggest the presence of notable differences in costs among utilities attributable to different weather conditions and locations. We have also obtained evidence as to the relationship between firms' inefficiency and some char- 
acteristics of their networks. In particular, most of our specifications suggest that larger networks tend to be more efficient than smaller ones, and that it is more difficult to manage firms with more numerous stations and overhead lines.

Based on the values of the estimated likelihood functions, we conclude that the NLC model is the preferred model, and that the results for the firms' technology and efficiency using the RSCFG, LCSF and ZISF models should be interpreted with caution. For instance, we have shown that our preferred model splits the sample into groups in rather different ways compared to more restrictive LCSF and ZISF models. Therefore, the efficiency scores in both LCSF and ZISF models are expected to be somewhat biased. In this sense, we have found that the efficiency scores of inefficient firms tend to be biased upwards if we do not distinguish between inefficient and fully efficient networks. The unobserved differences in technology could cause large biases in the estimated efficiency scores if they are ignored. On the other hand, our NLC model identifies a larger number of fully efficient firms than the ZISF model, indicating that some firms in the ZISF model are wrongly labelled as inefficient. In addition, other firms are wrongly labelled as fully efficient by the ZISF model.

From a policy point of view, both the large efficiency scores and the $40 \%$ of fully efficient firms found in our application suggest that Norwegian regulators have been able to encourage their networks to improve their performance during the sample period. For any regulator, our paper (including the simulation exercise) advocates prioritizing actions aiming to better fit firms' technology over actions aiming to control for (un)observed differences in performance. This obviously does not preclude that identifying differences in relative firms' efficiency would be still important to tune firms' incentives or to identify practical strategies to improve firms' efficiency.

\section{ACKNOWLEDGMENTS}

We would like to thank William Greene for his comments and support at various stages during the elaboration of the paper. A preliminary version of the paper can be found in Orea and Jamasb (2014). Thanks are also due to Guido Pepermans for his valuable comments to an earlier version of this paper. This research was partially funded by the Government of the Principality of Asturias and the European Regional Development Fund (ERDF).

\section{REFERENCES}

Alvarez, A., C. Amsler, L. Orea, and P. Schmidt (2006). "Interpreting and testing the scaling property in models where inefficiency depends on firm characteristics," Journal of Productivity Analysis 25(3): 201-212.

Caudill, S.B, and J.M. Ford (1993). "Biases in Frontier Estimation Due to Heteroscedasticity." Economic Letters 41: 1720. http://dx.doi.org/10.1016/0165-1765(93)90104-K.

Caudill, S.B., J.M. Ford and D.M. Gropper (1995). "Frontier Estimation and Firm-Specific Inefficiency Measures in the Presence of Heteroscedasticity." Journal of Business \& Economic Statistics 13: 105-111. http://dx.doi.org/10.1080/ 07350015.1995.10524583.

Cheng, X., E. Bjorndal and M.H. Bjorndal (2014). "Cost Efficiency Analysis Based on the DEA and StoNED Models: Case of Norwegian Electricity Distribution Companies." Norwegian School of Economics and Administration, Department of Business and Management Science Discussion Paper No. 2014/28. http://dx.doi.org/10.1109/eem.2014.6861260.

Cossent, R. (2013). Economic Regulation of Distribution System Operators and Its Adaptation to the Penetration of Distributed Energy Resources and Smart Grid Technologies, PhD Project, Escuela Técnica Superior de Ingeniería (ICAI), Madrid, mimeo.

Fried, H.O., C.A.K. Lovell and S.S. Schmidt (2008). The Measurement of Productive Efficiency and Productivity Growth, Oxford University Press. http://dx.doi.org/10.1093/acprof:oso/9780195183528.001.0001.

Grassetti, L. (2011). "A Novel Mixture Based Stochastic Frontier Model with Application to Hospital Efficiency.” Unpublished manuscript, University of Udine. 
Greene, W. (2005). "Reconsidering Heterogeneity in Panel Data Estimators of the Stochastic Frontier Model." Journal of Econometrics 126: 269-303. http://dx.doi.org/10.1016/j.jeconom.2004.05.003.

Growitsch, C., T. Jamasb and H. Wetzel (2012). "Efficiency Effects of Observed and Unobserved Heterogeneity: Evidence from Norwegian Electricity Distribution Networks." Energy Economics 34: 542-548. http://dx.doi.org/10.1016/ j.eneco.2011.10.013.

Haney, A.B. and M. Pollitt (2013). "International Benchmarking of Electricity Transmission by Regulators: A Contrast between Theory and Practice?." Energy Policy 62: 267-281. http://dx.doi.org/10.1016/j.enpol.2013.07.042.

Jamasb, T. and M. Pollitt (2001). "Benchmarking and Regulation: International Electricity Experience.” Utilities Policy 9(3): 107-130. http://dx.doi.org/10.1016/S0957-1787(01)00010-8.

Jamasb, T. and M. Söderberg (2010). "The Effects of Average Norm Model Regulation: The Case of Electricity Distribution in Sweden.” Review of Industrial Organization 36(3), May: 249-269. http://dx.doi.org/10.1007/s11151-010-9246-8.

Johnson, A.L. and Kuosmanen, T. (2012). "One-Stage and Two-Stage DEA Estimation of the Effects of Contextual Variables.” European Journal of Operational Research 220(2): 559-570. http://dx.doi.org/10.1016/j.ejor.2012.01.023.

Joskow, P. (2008). "Incentive Regulation and Its Application to Electricity Networks." Review of Network Industries 7(4): 547-560. http://dx.doi.org/10.2202/1446-9022.1161.

Kumbhakar, S.C., C.F. Parmeter and E.G. Tsionas (2013). “A Zero Inefficiency Stochastic Frontier Model.” Journal of Econometrics 172(1): 66-76. http://dx.doi.org/10.1016/j.jeconom.2012.08.021.

Kuosmanen, T. (2012). "Stochastic Semi-Nonparametric Frontier Estimation of Electricity Distribution Networks: Application of the StoNED Method in the Finnish Regulatory Model.” Energy Economics 34: 2189-2199. http://dx.doi.org/ 10.1016/j.eneco.2012.03.005.

Laffont, J.J. and J. Tirole (1993). A Theory of Incentives in Procurement and Regulation, MIT Press.

Llorca, M., L. Orea and M. Pollitt (2014). "Using the Latent Class Approach to Cluster Firms in Benchmarking: An Application to the US Electricity Transmission Industry." Operations Research Perspectives 1(1): 6-17. http://dx.doi.org/ 10.1016/j.orp.2014.03.002.

Miguéis, V.L., A.S. Camanho, E. Bjørndal and M. Bjørndal (2012). "Productivity Change and Innovation in Norwegian Electricity Distribution Companies." Journal of the Operational Research Society 63: 982-990. http://dx.doi.org/10.1057/ jors.2011.82.

Orea, L. and T. Jamasb (2014). "Identifying Efficient Regulated Firms with Unobserved Technological Heterogeneity: A Nested Latent Class Approach to Norwegian Electricity Distribution Networks.” Efficiency Series Paper 03/2014, Department of Economics, University of Oviedo.

Orea, L., T. Jamasb and Growitsch, C. (2015). "Using Supervised Environmental Composites in Production and Efficiency Analyses: An Application to Norwegian Electricity Networks.” Competition and Regulation in Network Industries 16(3): 256-283.

Orea, L. and S. Kumbhakar (2004). "Efficiency Measurement Using a Latent Class Stochastic Frontier Model." Empirical Economics 29: 169-183. http://dx.doi.org/10.1007/s00181-003-0184-2.

Reifschneider D. and R. Stevenson (1991). "Systematic Departures from the Frontier: A Framework for the Analysis of Firm Inefficiency.” International Economic Review 32: 715-723. http://dx.doi.org/10.2307/2527115.

Rho, S. and P. Schmidt (2015). “Are All Firms Inefficient?” Journal of Productivity Analysis 43(3): 327-349. http:// dx.doi.org/10.1007/s11123-013-0374-7.

Salvanes, K. and S. Tjøtta (1998). "A Test for Natural Monopoly with Application to Norwegian Electricity Distribution." Review of Industrial Organization 13(6): 669-685. http://dx.doi.org/10.1023/A:1007708912329.

Shleifer, A. (1985). "A Theory of Yardstick Competition." The RAND Journal of Economics 16(3): 319-327. http:// dx.doi.org/10.2307/2555560.

Silva, H. (2011). "Cost Efficiency in Periodic Tariff Reviews: The Reference Utility Approach and the Role of Interest Groups.” Public Utility Research Centre, University of Florida.

Stephens, M. (2000). "Dealing with Label Switching in Mixture Models." Journal of the Royal Statistical Society, Series B, Methodological, 62: 795-809. http://dx.doi.org/10.1111/1467-9868.00265.

Tsionas, E.G. (2002). "Stochastic Frontier Models with Random Coefficients.” Journal of Applied Econometrics 17(2): 127147. http://dx.doi.org/10.1002/jae.637. 


\section{APPENDIX A}

\section{Pooled Specification of the NLC Model}

This appendix develops a pooled specification of our NLC model that does not impose time-invariant class probabilities. There are not too many differences between the pooled and panel specification of the NLC model. Indeed, the cost frontier in the pooled specification is the same as in equation (1) and the probabilities of being inefficient and the technological-class probabilities are again modelled as two multinomial logit functions, as in equations (2) and (4). However, we now assume that they are firm and time specific:

$$
\begin{aligned}
& \prod_{i t \mid j}\left(\gamma_{j}\right)=\frac{\exp \left(\gamma_{j}^{\prime} z_{i t}\right)}{1+\exp \left(\gamma_{j}^{\prime} z_{i t}\right)} \\
& P_{i t j}\left(\delta_{j}\right)=\frac{\exp \left(\delta_{j}^{\prime} q_{i t}\right)}{1+\sum_{j=1}^{J-1} \exp \left(\delta_{j}^{\prime} q_{i t}\right)} \quad j=1, \ldots, J-1
\end{aligned}
$$

The pooled specification of the unconditional likelihood is also firm and time specific. It now can be written as:

$$
L F_{i t}(\theta, \delta)=\sum_{j=1}^{J}\left\{L F_{i t \mid j}^{S F} \prod_{i t \mid j}\left(\gamma_{j}\right)+L F_{i t \mid j}^{Z I}\left[1-\prod_{i t \mid j}\left(\gamma_{j}\right)\right]\right\} P_{i t j}\left(\delta_{j}\right)
$$

The overall likelihood function can be obtained once we add equation (1a) across firms and over time:

$$
\ln L F(\theta, \delta)=\sum_{i=1}^{N} \sum_{t=1}^{T} \ln L F_{i t}(\theta, \delta)
$$

\section{APPENDIX B}

\section{Simulation Exercise Details}

We consider a very simple data generating process: $y_{i t}=\alpha_{j}+\beta_{j} x_{i t}+v_{i t}+u_{i t}$, where $\alpha_{j}$ and $\beta_{j}$ are the technological parameters of technology $j(=1,2) ; \varepsilon_{i t}=v_{i t}+u_{i t}$ where $v_{i t} \sim N\left(0, \sigma_{v}^{2}\right)$ and $u_{i t} \sim N^{+}\left(0, \sigma_{u}^{2}\right)$ with probability $\mathrm{P}$ and $u_{i t}=0$ with probability 1 -P. We generate $x_{i t}$ from a $N(0,1)$. As the non-negative inefficiency term is added to the noise term, $y_{i t}$ can be interpreted as a cost variable. All our experiments are set so that $\sigma_{\varepsilon}^{2}=0.1$ in order to get a relatively large goodness-offit since most SFA applications use data from electricity distribution networks.

We carry out our simulations assuming that there are two different technologies in our data. They could differ only in the slope parameter (S-type scenarios), or in both the intercept and the slope parameter (L-type scenarios). In S-type scenarios, we assume that $\alpha_{1}=\alpha_{2}=10, \beta_{1}=1$ and $\beta_{2}=0.5$. In L-type scenarios, we assume that $\alpha_{2}=\alpha_{1}+0.5$. We consider two possible values for the proportion of inefficient firms, $\mathrm{P}=0.6$ and $\mathrm{P}=0.8$. In the first case, we assume that $\lambda=2$. In the second case, in addition to $\lambda=2$, we also assume a lesser value for $\lambda$ (i.e. $\lambda=1$ ) in order to examine the role of larger inefficiencies in our data. In summary, our simulation exercise involves the following six scenarios: 


\begin{tabular}{|c|c|c|c|c|c|}
\hline \multicolumn{2}{|c|}{1} & \multicolumn{2}{|c|}{2} & \multicolumn{2}{|c|}{3} \\
\hline S-type & L-type & S-type & L-type & S-type & L-type \\
\hline \multicolumn{6}{|c|}{$\begin{array}{c}\beta_{1}=1 \\
\beta_{2}=0.5\end{array}$} \\
\hline $\begin{array}{l}\alpha_{1}=10 \\
\alpha_{2}=10\end{array}$ & $\begin{array}{c}\alpha_{1}=10 \\
\alpha_{2}=10.5\end{array}$ & $\begin{array}{l}\alpha_{1}=10 \\
\alpha_{2}=10\end{array}$ & $\begin{array}{c}\alpha_{1}=10 \\
\alpha_{2}=10.5\end{array}$ & $\begin{array}{l}\alpha_{1}=10 \\
\alpha_{2}=10\end{array}$ & $\begin{array}{c}\alpha_{1}=10 \\
\alpha_{2}=10.5\end{array}$ \\
\hline \multicolumn{2}{|c|}{$\lambda=1$} & \multicolumn{4}{|c|}{$\lambda=2$} \\
\hline \multicolumn{4}{|c|}{$\mathrm{P}=0.8$} & \multicolumn{2}{|c|}{$\mathrm{P}=0.6$} \\
\hline
\end{tabular}

For all the replications we consider that $\mathrm{N}=100$ and $\mathrm{T}=5$ in each technology-class. Therefore, the total number of observations in replication is 1,000 . In order to identify the observations that are fully efficient in each technology, we generate 1,000 values from a uniform distribution and set $u_{i t}=0$ in those observations where the generated value is larger than P. All of our simulations involve estimating four competing models (Basic SFA, ZISF, LCSF, and NLC). All of them are based on 500 replications. This implies that our simulation exercise requires estimating about 12,000 models. We maximize the likelihood by a direct optimization with respect to all of the parameters using GAUSS.

The mean, bias, and MSE of the technological parameter estimates are shown below:

\begin{tabular}{|c|c|c|c|c|c|c|c|c|c|}
\hline \multirow[b]{2}{*}{ Model } & \multirow[b]{2}{*}{ Coef. } & \multicolumn{4}{|c|}{ Scenario S1 } & \multicolumn{4}{|c|}{ Scenario L1 } \\
\hline & & value & mean & bias & MSE & value & mean & bias & MSE \\
\hline \multirow[t]{4}{*}{ Basic SFA } & $\alpha_{1}$ & 10 & 9.964 & -0.036 & 0.009 & 10 & 10.173 & 0.173 & 0.053 \\
\hline & $\beta_{1}$ & 1 & 0.750 & -0.250 & 0.063 & 1 & 0.765 & -0.235 & 0.056 \\
\hline & $\alpha_{2}$ & 10 & 9.964 & -0.036 & 0.009 & 10.5 & 10.173 & -0.327 & 0.129 \\
\hline & $\beta_{2}$ & 0.5 & 0.750 & 0.250 & 0.063 & 0.5 & 0.765 & 0.265 & 0.071 \\
\hline \multirow[t]{4}{*}{ ZISF } & $\alpha_{1}$ & 10 & 10.027 & 0.027 & 0.007 & 10 & 10.175 & 0.175 & 0.031 \\
\hline & $\beta_{1}$ & 1 & 0.750 & -0.250 & 0.063 & 1 & 0.813 & -0.187 & 0.035 \\
\hline & $\alpha_{2}$ & 10 & 10.027 & 0.027 & 0.007 & 10.5 & 10.175 & -0.325 & 0.106 \\
\hline & $\beta_{2}$ & 0.5 & 0.750 & 0.250 & 0.063 & 0.5 & 0.813 & 0.313 & 0.098 \\
\hline \multirow[t]{4}{*}{ LCSF } & $\alpha_{1}$ & 10 & 9.967 & -0.033 & 0.009 & 10 & 9.974 & -0.026 & 0.009 \\
\hline & $\beta_{1}$ & 1 & 1.001 & 0.001 & 0.000 & 1 & 0.998 & -0.002 & 0.000 \\
\hline & $\alpha_{2}$ & 10 & 9.974 & -0.026 & 0.008 & 10.5 & 10.485 & -0.015 & 0.009 \\
\hline & $\beta_{2}$ & 0.5 & 0.499 & -0.001 & 0.000 & 0.5 & 0.501 & 0.001 & 0.000 \\
\hline \multirow[t]{4}{*}{ NLC } & $\alpha_{1}$ & 10 & 10.008 & 0.008 & 0.004 & 10 & 10.001 & 0.001 & 0.004 \\
\hline & $\beta_{1}$ & 1 & 1.001 & 0.001 & 0.000 & 1 & 1.000 & 0.000 & 0.000 \\
\hline & $\alpha_{2}$ & 10 & 10.005 & 0.005 & 0.004 & 10.5 & 10.521 & 0.021 & 0.006 \\
\hline & $\beta_{2}$ & 0.5 & 0.500 & -0.000 & 0.000 & 0.5 & 0.499 & -0.001 & 0.000 \\
\hline
\end{tabular}


Scenario S2

$\left(\lambda=2, \mathrm{P}=0.8, \alpha_{1}=\alpha_{2}, \beta_{1} \neq \beta_{2}\right)$
Scenario L2

$\left(\lambda=2, P=0.8, \alpha_{1} \neq \alpha_{2}, \beta_{1} \neq \beta_{2}\right)$

\begin{tabular}{|c|c|c|c|c|c|c|c|c|c|}
\hline Model & Coef. & value & mean & bias & MSE & value & mean & bias & MSE \\
\hline \multirow[t]{4}{*}{ Basic SFA } & $\alpha_{1}$ & 10 & 9.919 & -0.081 & 0.008 & 10 & 10.071 & 0.071 & 0.018 \\
\hline & $\beta_{1}$ & 1 & 0.750 & -0.250 & 0.063 & 1 & 0.797 & -0.203 & 0.042 \\
\hline & $\alpha_{2}$ & 10 & 9.919 & -0.081 & 0.008 & 10.5 & 10.071 & -0.429 & 0.196 \\
\hline & $\beta_{2}$ & 0.5 & 0.750 & 0.250 & 0.063 & 0.5 & 0.797 & 0.297 & 0.090 \\
\hline \multirow[t]{4}{*}{ ZISF } & $\alpha_{1}$ & 10 & 10.005 & 0.005 & 0.002 & 10 & 10.233 & 0.233 & 0.055 \\
\hline & $\beta_{1}$ & 1 & 0.750 & -0.250 & 0.063 & 1 & 0.817 & -0.183 & 0.034 \\
\hline & $\alpha_{2}$ & 10 & 10.005 & 0.005 & 0.002 & 10.5 & 10.233 & -0.267 & 0.072 \\
\hline & $\beta_{2}$ & 0.5 & 0.750 & 0.250 & 0.063 & 0.5 & 0.817 & 0.317 & 0.101 \\
\hline \multirow[t]{4}{*}{ LCSF } & $\alpha_{1}$ & 10 & 9.911 & -0.089 & 0.009 & 10 & 9.910 & -0.090 & 0.009 \\
\hline & $\beta_{1}$ & 1 & 0.999 & -0.001 & 0.000 & 1 & 0.996 & -0.004 & 0.000 \\
\hline & $\alpha_{2}$ & 10 & 9.915 & -0.085 & 0.008 & 10.5 & 10.421 & -0.079 & 0.007 \\
\hline & $\beta_{2}$ & 0.5 & 0.501 & 0.001 & 0.000 & 0.5 & 0.503 & 0.003 & 0.000 \\
\hline \multirow[t]{4}{*}{ NLC } & $\alpha_{1}$ & 10 & 10.000 & 0.000 & 0.001 & 10 & 9.999 & -0.001 & 0.001 \\
\hline & $\beta_{1}$ & 1 & 0.999 & -0.001 & 0.000 & 1 & 1.000 & 0.000 & 0.000 \\
\hline & $\alpha_{2}$ & 10 & 10.002 & 0.002 & 0.001 & 10.5 & 10.499 & -0.001 & 0.001 \\
\hline & $\beta_{2}$ & 0.5 & 0.500 & -0.000 & 0.000 & 0.5 & 0.501 & 0.001 & 0.000 \\
\hline & & \multicolumn{4}{|c|}{ io $\mathrm{S3}$} & \multicolumn{4}{|c|}{ io $\mathrm{L3}$} \\
\hline
\end{tabular}

\begin{tabular}{|c|c|c|c|c|c|c|c|c|c|}
\hline Model & Coef. & value & mean & bias & MSE & value & mean & bias & MSE \\
\hline \multirow[t]{4}{*}{ Basic SFA } & $\alpha_{1}$ & 10 & 9.835 & -0.165 & 0.029 & 10 & 9.959 & -0.041 & 0.009 \\
\hline & $\beta_{1}$ & 1 & 0.750 & -0.250 & 0.063 & 1 & 0.812 & -0.188 & 0.036 \\
\hline & $\alpha_{2}$ & 10 & 9.835 & -0.165 & 0.029 & 10.5 & 9.959 & -0.541 & 0.301 \\
\hline & $\beta_{2}$ & 0.5 & 0.750 & 0.250 & 0.062 & 0.5 & 0.812 & 0.312 & 0.098 \\
\hline \multirow{4}{*}{ ZISF } & $\alpha_{1}$ & 10 & 10.000 & 0.000 & 0.001 & 10 & 10.125 & 0.125 & 0.017 \\
\hline & $\beta_{1}$ & 1 & 0.750 & -0.250 & 0.063 & 1 & 0.831 & -0.169 & 0.029 \\
\hline & $\alpha_{2}$ & 10 & 10.000 & 0.000 & 0.001 & 10.5 & 10.125 & -0.375 & 0.142 \\
\hline & $\beta_{2}$ & 0.5 & 0.750 & 0.250 & 0.063 & 0.5 & 0.831 & 0.331 & 0.110 \\
\hline \multirow[t]{4}{*}{ LCSF } & $\alpha_{1}$ & 10 & 9.841 & -0.159 & 0.026 & 10 & 9.841 & -0.159 & 0.026 \\
\hline & $\beta_{1}$ & 1 & 1.001 & 0.001 & 0.000 & 1 & 0.998 & -0.002 & 0.000 \\
\hline & $\alpha_{2}$ & 10 & 9.839 & -0.161 & 0.026 & 10.5 & 10.344 & -0.156 & 0.025 \\
\hline & $\beta_{2}$ & 0.5 & 0.500 & 0.000 & 0.000 & 0.5 & 0.505 & 0.005 & 0.000 \\
\hline \multirow[t]{4}{*}{ NLC } & $\alpha_{1}$ & 10 & 10.002 & 0.002 & 0.000 & 10 & 10.000 & 0.000 & 0.000 \\
\hline & $\beta_{1}$ & 1 & 1.000 & 0.000 & 0.000 & 1 & 1.001 & 0.001 & 0.000 \\
\hline & $\alpha_{2}$ & 10 & 10.000 & -0.000 & 0.000 & 10.5 & 10.502 & 0.002 & 0.000 \\
\hline & $\beta_{2}$ & 0.5 & 0.500 & 0.000 & 0.000 & 0.5 & 0.500 & 0.000 & 0.000 \\
\hline
\end{tabular}




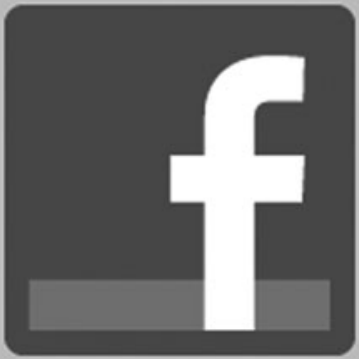

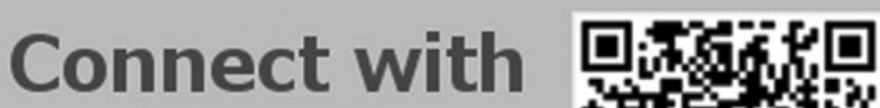

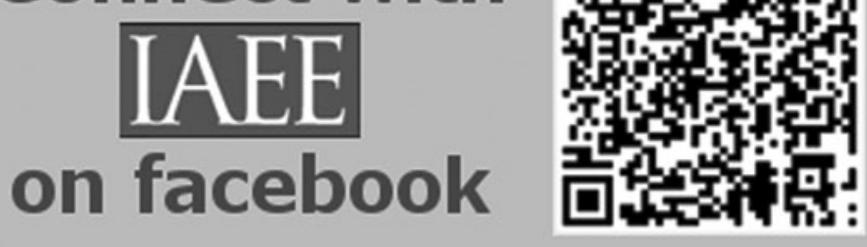

